\title{
Homotopy for small multifunctions $\left({ }^{*}\right)$
}

by

\section{Helga Schirmer (Ottawa)}

Abstract. A multifunction $\varphi: X \rightarrow S^{n}$ from a space $X$ into an $n$-sphere $S^{n}$ is called small if each point-image $\varphi(x)$ is contained in a semi-sphere. A small homotopy is a multifunction of the form $\Phi: X \times I \rightarrow S^{n}$, where $I$ denotes the unit interval. It is shown that every usc resp. lse small multifunction is related to a single-valued map by an usc resp. lsc small homotopy. Applications are given to multi-homotopy groups of spheres, and to multi-vector fields on spheres.

1. Introduction. A multifunction $\varphi: X \rightarrow Y$ from a topological space $X$ into a topological space $X$ is a correspondence which assigns to each point $x \in X$ a non-empty subset $\varphi(x)$ of $Y$. We call $\varphi$ usc (upper semicontinuous) if for every open set $V \subset Y$ with $\varphi(x) \subset V$ there exists an open neighbourhood $U$ of $x$ such that $\varphi(U) \subset V$, and if $\varphi(x)$ is closed for all $x \in X$. (Current usage often omits the condition that an usc multifunction should be point-closed, but it is needed in the proof of Lemma 2.5.) We call $\varphi$ lsc (lower semi-continuous) if for every $x \in X$ and every open $V \subset Y$ with $\varphi(x) \frown V \neq \varnothing$ there exists an open neighbourhood $U$ of $x$ such that $\varphi\left(x^{\prime}\right) \cap \nabla \neq \emptyset$ for all $x^{\prime} \in U$. The term map is reserved for single-valued continuous functions.

Several authors have considered the problem whether any usc multifunction $\varphi: X \rightarrow S^{n}$ whose values $\varphi(x)$ are proper subsets of an $n$-sphere $S^{n}$ is homotopic to a map under a suitably restricted homotopy. Acyclic multifunctions were e.g. investigated by J. W. Jaworowski [5], but it is still not known whether any usc acyclic multifunction $\varphi: X \rightarrow S^{n}$ is acyclically homotopic to a map. T. R. Brahana, M. K. Fort, Jr., and W. G. Horstman [2] proved that every usc cellular multifunction from a finite-dimensional compact metric space $X$ into $S^{n}$ can be transformed into a map by a cellular homotopy.

Here we derive similar results for "small" multifunctions. Define a small cap of the $n$-sphere $\|x\|=1$ in $E^{n+1}$ as the smaller part cut off by a hyperplane of distance $d$ (where $0<d<1$ ) from the centre, and

$\left(^{*}\right)$ This research was partially supported by the National Research Council of Canada (Grant A 7579). 
call a multifunction $\varphi: X \rightarrow S^{n}$ small if each $\varphi(x)$ is contained in a small cap, where the cap can vary with $x$. (These multifunctions are $\delta$-small, with $\delta=\pi$, in the sense of [8], [9], as their spherical diameter is $<\pi$.) A small homotopy is a small multifunction of the form $\Phi: X \times I \rightarrow S^{n}$.

We show that every usc small multifunction $\varphi: X \rightarrow S^{n}$ is related to a map by a small homotopy (Theorem 2.6). This is done by constructing, with the help of the "spherical convex hull" (see $\S 2)$ of $\varphi(x)$, an usc cellular multifunction associated with $\varphi$, and then using results for such multifunctions from [2]. We also prove a similar result for lsc small multifunctions (Theorem 3.3). In this case it follows from a theorem by E. Michael [6] that there exists a map $f: X \rightarrow S^{n}$ for which $f(x)$ is contained in the spherical convex hull of $\varphi(x)$. This map therefore approximates a selection, and is homotopic to $\varphi$.

Isomorphisms between the ordinary homotopy groups of spheres and those arising from use or lsc small multifunctions can be obtained in a routine way $(\S 4)$. Note that no condition related to acyclicity or even connectedness is imposed on $\varphi(x)$. But an example at the end of $\S 4$ shows that the results are no longer true if $\varphi(x)$ is an arbitrary proper subset of $S^{n}$.

We conclude with an application to small multi-vector fields on spheres and show that they are homotopic to single-valued ones ( $\$ 5$ ).

All results are stated under the assumption that $X$ is a finite-dimensional compact metric space, although those of $\S 3$ are actually true under the weaker assumption that $X$ is $T_{1}$ and collection-wise normal (see [6], p. 380). Background material on multifunctions can e.g. be found in [1], on convexity in [4] or [11].

2. Homotopy for usc small multifunctions. The aim of this paragraph is Theorem 2.6 below, in which we show that every usc small multifunction $\varphi: X \rightarrow S^{n}$ is related to a map by a small homotopy. The proof uses the concept of the spherical convex. hull, which we consider first.

Call a subset $A$ of $S^{n}$ spherically convex (or weakly convex, see [3], p. 157) if it contains, with each pair of its points, the shortest arc or a semicircular arc of a great circle determined by them. Define the spherical convex hull (written $s \operatorname{con} v A$ ) as the intersection of all spherically convex sets containing $A$. Let $a_{1}, a_{2}, \ldots, a_{m}$ (where $m \leqslant n+1$ ) be points of $S^{n}$ which span an $m$-dimensional simplex $\sigma=\sigma\left(a_{1}, a_{2}, \ldots, a_{m}\right)$ in an $(n+1)$ dimensional Euclidean space $E^{n+1}$ containing $S^{n}$. If the set $\left\{a_{1}, a_{2}, \ldots, a_{m}\right\}$ is small, then the origin $0 \notin \sigma\left(a_{1}, a_{2}, \ldots, a_{m}\right)$, and the image $s$ $=s\left(a_{1}, a_{2}, \ldots, a_{m}\right)$ of $\sigma\left(a_{1}, a_{2}, \ldots, a_{m}\right)$ under the projection $p: E^{n+1} \backslash\{0\} \rightarrow S^{n}$ from the origin given by $p(x)=x /\|x\|$ is called a spherical simplex. It is well-known that the convex hull conv $A$ of a subset $A$ of $E^{n+1}$ is the union of all simplices whose vertices are contained in $A$. We need an analogon for the spherical convex hull.

Lenvid 2.1. Let $A$ be a small subset of $\mathbb{S}^{n}$. Then sconv $A$ is the union of all spherical simplices whose vertices are contained in $A$.

Proof. Let $A^{*}$ denote the union of all spherical simplices whose vertices are in $A$.

(i) $A^{*} C$ sconv $A$ : It follows easily by induction on the number of vertices (as in the case of conv $A$ in $E^{n+1}$ ) that each spherical simplex $s\left(a_{1}, a_{2}, \ldots, a_{m}\right)$, with $a_{1}, a_{2}, \ldots, a_{m} \in A$, is contained in sconv $A$, hence $A^{*} C \operatorname{sconv} A$.

(ii) $\operatorname{sconv} A \subset A^{*}$ : As $s=s(a) \subset A^{*}$ for all $a \in A$, we have $A \subset A^{*}$. Therefore sconv $A \subset A^{*}$ is true if we can show that $A^{*}$ is spherically convex. For this purpose, take any two points $x, y \in A^{*}$. Then $x \in s\left(a_{1}, a_{2}, \ldots, a_{r}\right)$ and $y \in s\left(b_{1}, b_{2}, \ldots, b_{s}\right)$, where $A_{1}=\left\{a_{1}, a_{2}, \ldots, a_{r}, b_{1}, b_{2}, \ldots, b_{s}\right\}$ is a subset of $A$. If $z$ is any point in the shortest are from $x$ to $y$, then we can choose $x^{\prime} \in \sigma\left(a_{1}, a_{2}, \ldots, a_{r}\right)$ and $y^{\prime} \in \sigma\left(b_{1}, b_{2}, \ldots, b_{s}\right)$ such that $x=p\left(x^{\prime}\right)$ and $y=p\left(y^{\prime}\right)$, and choose $z^{\prime}$ in the line segment from $x^{\prime}$ to $y^{\prime}$ such that $z=p\left(z^{\prime}\right)$. As $z^{\prime} \in \operatorname{conv} A_{1}$, there exists a simplex $\sigma=\sigma\left(c_{1}, c_{2}, \ldots, c_{t}\right)$ with vertices in $A_{1}$ which contains $z^{\prime}$. Then

$$
z \in p\left[\sigma\left(c_{1}, c_{2}, \ldots, c_{t}\right)\right]=s\left(c_{1}, c_{2}, \ldots, c_{t}\right),
$$

so that $z \in A_{z}^{*}$. Therefore $A^{*}$ is spherically convex.

Lenina 2.2. If $A$ is a small subset of $S^{n}$, then $p(\operatorname{conv} A)=\operatorname{sconv} A$.

Proof. As conv $A$ is the union of all simplices with vertices in $A$, this follows immediately from Lemma 2.1.

A subset $A$ of $S^{n}$ is called cellutar if there exists a sequence $E_{1} \supset E_{2}$ $\supset E_{3} \supset \ldots$ of topological $n$-cells in $S^{n}$ such that $A=\bigcap_{k=1}^{\infty} E_{k}$ and $A \subset \operatorname{Int} E_{k}$ for all $k$.

Lewra 2.3. If $A$ is a small closed set, then sconvA is cellular.

Proof. As $A$ is small, $0 \notin \operatorname{conv} A$, and as conv $A$ is closed, we can select a sequence of positive numbers $\varepsilon_{1}>\varepsilon_{2}>\varepsilon_{3}>\ldots$ converging to zero such that $0 \notin \bar{N}\left(\operatorname{conv} A, \varepsilon_{k}\right)$, where

$$
\left.\bar{N}\left(\operatorname{con} \nabla A, \varepsilon_{k}\right)=\left\{x^{\prime} \in E^{n+1}\right\}\left\|x-x^{\prime}\right\| \leqslant \varepsilon_{k} \text { for some } x \in \operatorname{conv} A\right\} .
$$

The set $\bar{N}\left(\operatorname{conv} A, \varepsilon_{k}\right)$ is closed and convex, and hence $C_{k}=\bar{N}\left(\operatorname{conv} A, \varepsilon_{k}\right) \cap$ $\cap B^{n+1}$ is closed and convex also. The definition of spherically convex implies that the closed set $E_{k}=p\left(C_{k}\right)$ is spherically convex. Similar to the Euclidean case it follows that $E_{k}$ is homeomorphic to a closed ball, and as $E_{k}$ is $n$-dimensional by construction, it is a topological $n$-cell. We further have conv $A \subset \operatorname{Int} C_{k}$, and as $p$ is an open map, Lemma 2.2 
yields sconv $A \subset \operatorname{Int} p\left(C_{k}\right)=\operatorname{Int} E_{k}$. It is also true that $\operatorname{sconv} A=\bigcap_{k=1}^{\infty} E_{k}$. Therefore the set $\operatorname{scon} \nabla A$ is cellular.

Propostrion 2.4. Let $\varphi: X \rightarrow S^{n}$ be an usc small multifunction from a finite-dimensional compact metric space $X$ into an $n$-sphere. Then there exists a map $g: X \rightarrow S^{n}$ with $g(x) \in S^{m} \backslash \varphi(x)$ for all $x \in X$.

Proof. Define a multifunction $\chi: X \rightarrow E^{n+1}$ by $\chi(x)=\operatorname{con} \overline{\varphi(x)}$, where $\overline{\varphi(x)}$ denotes the closure of $\varphi(x)$. We first show that $\chi$ is usc. Take any $x \in X$, and let $V$ be an open subset of $E^{n+1}$ with $\chi(x) \subset V$. Choose $\varepsilon>0$ such that $N(\chi(x), \varepsilon) \subset V$. As $\varphi$ is usc, there exists an open set $C=U(x)$ containing $x$ with $\varphi(U) \subset N(\varphi(x), \varepsilon / 2)$. Now take any $x^{\prime} \in U$. If $y^{\prime} \in \chi\left(x^{\prime}\right)$, then $y^{\prime}$ is contained in a simplex $\sigma=\sigma\left(a_{1}^{\prime}, a_{2}^{\prime}, \ldots, a_{m}^{\prime}\right)$ with $a_{i}^{\prime} \in \overline{\varphi\left(x^{\prime}\right)}$ for $i=1,2, \ldots, m$, so that

$$
y^{\prime}=\sum_{i=1}^{m} \lambda_{i} a_{i}^{\prime} \quad \text { with } \sum_{i=1}^{m} \lambda_{i}=1 \text { and all } \lambda_{i} \geqslant 0 .
$$

As $\varphi\left(x^{\prime}\right) \subset N(\varphi(x), \varepsilon / 2)$, we can select points $a_{1}, a_{2}, \ldots, a_{m}$ in $\varphi(x)$ with $\left\|a_{i}-a_{i}^{\prime}\right\|<\varepsilon$. If $y=\sum_{i=1}^{m} \lambda_{i} a_{i}$, then

$$
\left\|y-y^{\prime}\right\| \leqslant \sum_{i=1}^{m} \lambda_{i}\left\|a_{i}-a_{i}^{\prime}\right\|<\varepsilon .
$$

As $y \in \operatorname{conv} \varphi(x) \subset \chi(x)$, we have $y^{\prime} \epsilon N(\chi(x), \varepsilon) \subset V$ and hence $\chi(U) \subset V$. So $\chi$ is usc.

As $\varphi(x)$ is small, we have conv $\overline{\varphi(x)} \subset B^{n+1} \backslash\{0\}$, and therefore a small multifunction $\psi: X \rightarrow S^{n}$ can be defined by $\psi(x)=p \circ \chi(x)=\operatorname{sconv} \overline{\varphi(x)}$. The composite of two usc multifunctions is usc, and as conv $\overline{\varphi(x)}$ is closed, so is $\psi(x)$. Lemma 2.3 shows that $\psi(x)$ is cellular, and hence it follows from [2], Theorem 1 that there exists a map $f: X \rightarrow S^{n}$ such that $f(x)$ $\in S^{m} \backslash \psi(x)$ for all $x \in X$.

We call an usc (lsc) small multifunction $\Phi: X \times I \rightarrow S^{n}$ an usc (lsc) small homotopy between the multifunctions $\varphi_{0}(x)=\Phi(x, 0)$ and $\varphi_{1}(x)$ $=\Phi(x, 1)$. As in [2] we call it a special homotopy if in addition $\Phi(x, t)$ is homeomorphic to $\Phi(x, 0)$ for all $x \in X$ and $0 \leqslant t<1$. Denote the antipodal point of $y$ in $S^{n}$ by $-y$.

Lemira 2.5. Let $\varphi: X \rightarrow S^{n}$ be an usc (1sc) small multifunction and $f: X \rightarrow S^{n}$ be a map such that $-f(x) \notin \varphi(x)$ for all $x \in X$. Then there exists a special homotopy between $\varphi$ and $f$.

Proof. As in the proof of [2], Lemma 3 a homotopy can be defined by

$$
\Phi(x, t)=\left\{J_{-f(x)}(y, t) \mid y \in \varphi(x)\right\}
$$

where for each $a \in S^{n}$ the map $J_{a}:\left[S^{n} \backslash\{a\}\right] \times I \rightarrow S^{n}$ is given by $J_{a}(x, t)=[-t a+(1-t) x] /\|-t a+(1-t) x\|$ for $x \in S^{n} \backslash\{a\}$ and $0 \leqslant t \leqslant 1$. The verification that $\Phi$ is special is easy.

THEOREM 2.6. Let $\varphi: X \rightarrow S^{n}$ be an usc small multifunction from a finite-dimensional compact metric space $X$ into an $n$-sphere. Then there exists a special homotopy between $\varphi$ and a map $f: X \rightarrow S^{n}$.

Proof. From Proposition 2.4 and Lemma 2.5, with $f=-g$.

3. Homotopy for Isc small multifunctions. We now prove the analogon of Theorem 2.6 for lsc small multifunctions. But the method is different; instead of Proposition 2.4 we use the following Proposition 3.1 which is a consequence of a selection theorem by E. Michael.

Proposirion 3.1. Let $X$ be a finite-dimensional compact metric space and $\varphi: X \rightarrow S^{n}$ be a lsc small multifunction. Then there exists a map $f: X \rightarrow S^{n}$ such that $f(x) \in \operatorname{sconv} \varphi(x)$ for all $x \in X$.

Proof. Define a multifunction $\chi: X \rightarrow E^{n+1}$ by $\chi(x)=\operatorname{con} \nabla \varphi(x)$. As in the proof of Proposition 2.4 it is easy to show that $\chi$ is lse (see also [6], Proposition 2.6). It now follows from [6], Theorem 3.1'" that there exists a continuous selection $g: X \rightarrow E^{n+1}$ of $\chi$ (i.e. a map such that $g(x) \in \chi(x)$ for all $x \in X)$. As the closed $(n+1)$-ball $B^{n+1}$ bounded by $S^{n}$ is convex and as $\varphi(x)$ is small, we actually have $\chi(x) \subset B^{n+1} \backslash\{0\}$ and hence $g(x)$ $\epsilon B^{n+1} \backslash\{0\}$. Define a map $f: X \rightarrow S^{n}$ by $f=p \circ g$, where $p$ is again the projection from the origin. As $g(x) \epsilon \operatorname{conv} \varphi(x)$, Lemma 2.2 implies $f(x)$ $\epsilon \operatorname{sconv} \varphi(x)$, and Proposition 3.1 is proved.

The following selection theorem is an immediate consequence of Proposition 3.1.

COROLIARY 3.2. Let $\varphi: X \rightarrow S^{n}$ be a lse small multifunction from a finite-dimensional compact metric space $X$ into an $n$-sphere for which each $\varphi(x)$ is spherically convex. Then $\varphi$ has a continuous selection.

THEOREM 3.3. Let $\varphi: X \rightarrow S^{n}$ be a lsc small multifunction from a finitedimensional compact metric space $X$ into an $n$-sphere. Then there exists a special homotopy between $\varphi$ and a map $f: X \rightarrow S^{n}$.

Proof. From Proposition 3.1 we obtain a map $g: X \rightarrow S^{n}$ such that $g(x) \in \operatorname{sconv} \varphi(x)$ for all $x \in X$. As $\varphi(x)$ is small it follows that $-g(x)$ $\notin \operatorname{sconv} \varphi(x)$ and hence $-g(x) \notin \varphi(x)$. Therefore the map $f: X \rightarrow S^{n}$ defined by $f(x)=-g(x)$ is related to $\varphi$ by a special homotopy according to Lemma 2.5.

4. Homotopy groups of small multifunctions. It has been shown by C. J. Rhee [7] that the homotopy group arising from cellular homotopy 
classes of usc cellular multifunctions of the form $\left(I^{m}, \mathrm{Bd} I^{m}\right) \rightarrow\left(S^{n}, a\right)$ in the standard way is isomorphic to the ordinary homotopy group $\pi_{m}\left(S^{n}\right)$. The crucial steps in the construction of the isomorphism are the analogue of Theorem 2.6 for cellular multifunctions contained in [2], and the fact that two maps $f_{0}, f_{1}: X \rightarrow S^{n}$ related by a cellular homotopy are also related by a single-valued homotopy [2]. This is still true for small homotopies, as the next theorem can be proved in a way completely analogous to Theorem 3 in [2].

THEOREM 4.1. If $X$ is a finite-dimensional compact metric space and $f_{0}, f_{1}: X \rightarrow S^{n}$ are maps related by an usc or lse small homotopy, then they are also related by a single-valued homotopy.

Multi-homotopy groups for small multifunctions can be defined as in [10]. Denote by $M_{u} \Pi_{m}\left(S^{n}, a\right)$ the homotopy group formed from the set of use small multifunctions of the form $\left(I^{m}, \mathrm{Bd} I^{m}\right) \rightarrow\left(S^{n}, a\right)$ under usc small homotopies, and define $M_{l} \Pi_{m}\left(S^{n}, a\right)$ for lsc small multifunctions correspondingly. The methods used in [7] carry over to verify the following: theorem; details are omitted.

THEOREM 4.2. The groups $\boldsymbol{M}_{u} \Pi_{m}\left(S^{n}, a\right)$ and $M_{l} \Pi_{m}\left(S^{n}, a\right)$ are both isomorphic to $\pi_{m}\left(S^{n}\right)$.

Remark. It is possible that the definition of "small" used in this paper can be relaxed. But the following example shows that a condition which only asks that each $\varphi(x)$ is a proper subset of $S^{n}$ leads to difficulties.

ExAMPLE. Define a homotopy $\Phi: S^{2} \times I \rightarrow S^{2}$ by

$$
\Phi(x, t)=\left\{y \in S^{2} \mid \varrho(x, y)=t \pi\right\} \quad \text { for } \quad x \in S^{2}, 0 \leqslant t \leqslant 1 .
$$

Then $\Phi$ is a continuous (i.e. both use and lsc) multifunction, and each $\Phi(x, t)$ is a proper subset of $S^{2}$. But it is not small, as $\Phi\left(x, \frac{1}{2}\right)$ is not small. As $\Phi(x, 0)$ is the identity and $\Phi(x, 1)$ is the antipodal map, we see that Theorem 4.1, and hence Theorem 4.2, are not true any more if "small" is replaced by "proper subset of $S^{n \text { " }}$.

5. Small multi-vector fields on spheres. A (single-valued) vector field on a sphere $S^{n}(n \geqslant 1)$ is a map $f: S^{n} \rightarrow S^{n}$ such that for every $x \in S^{n}$ the vectors $\overrightarrow{O x}$ and $\overrightarrow{O f}(x)$ are orthogonal. Similarly we define a multi-vector field on $S^{n}$ as a multifunction $\varphi: S^{n} \rightarrow S^{n}$ such that for every $x \in S^{n}$ the vector $\overrightarrow{O x}$ is orthogonal to $\overrightarrow{O y}$ for all $y \in \varphi(x)$. The multi-vector field is called usc (lsc) if the function $\varphi$ is use (lsc). It is called small if $\varphi$ is small. Two use (lsc) small multi-vector fields $\varphi_{0}, \varphi_{1}: S^{n} \rightarrow S^{n}$ are called homotopic if there exists an use (lsc) small homotopy $\Phi: S^{n} \times I \rightarrow S^{n}$ such that $\Phi(x, 0)$ $=\varphi_{0}(x), \Phi(x, 1)=\varphi_{1}(x)$, and $\overrightarrow{O x}$ is orthogonal to $\overrightarrow{O y}$ for all $y \in \Phi(x, t)$ and $0 \leqslant t \leqslant 1$.
THEOREM 5.1. Every use or lsc small multi-vector field on $S^{n}(n \geqslant 1)$ is homotopic to a single-valued vector field.

Proof. (i) Let $\varphi: S^{n} \rightarrow S^{n}$ be an use small multi-vector field. Denote by $\operatorname{Con}(A, x)$ the cone in $E^{n+1}$ over $A \subset E^{n+1}$ with vertex $x$ (i.e. the join of $A$ and $x)$, and define for erery $x \in S^{n}$ the set $\psi(x) \subset E^{n+1}$ by

$$
\psi(x)=\operatorname{Con}(\operatorname{sen} \nabla \overline{\varphi(x)}, x) \cup \operatorname{Con}(\operatorname{seonv} \overline{\varphi(x)},-x) .
$$

As $\varphi$ is small, $0 \notin \psi(x)$, and hence a multifunction $\chi: S^{n} \rightarrow S^{n}$ can be defined by $\chi(x)=p(\psi(x))$, where $p: E^{n+1} \backslash\{0\} \rightarrow S^{n}$ is again the projection from the origin.

It was shown in the proof of Proposition 2.4 that the multifunction $\operatorname{sconv} \overline{\varphi(x)}$ is usc, and a very similar argument shows that $\psi: S^{n} \rightarrow E^{n+1} \backslash\{0\}$ is usc also. Therefore $\chi: S^{n} \rightarrow S^{n}$ is use.

Each $\chi(x)$ is cellular: As $\varphi(x)$ is small, we can select a sequence of positive numbers $\varepsilon_{1}>\varepsilon_{2}>\varepsilon_{3}>\ldots$ such that $0 \notin \bar{N}\left(\operatorname{Con}(A, x), \varepsilon_{k}\right)$, where $A=\operatorname{sconv} \overline{\varphi(x)}$ and $k=1,2,3 \ldots$ Cut $B^{n+1}$ into two parts by the hyperplane through 0 and orthogonal to $\overrightarrow{O x}$, and let $B_{+}$be the (closed) part containing $x$. Then $\bar{N}\left(\operatorname{Con}(A, x), \varepsilon_{k}\right) \cap B_{+}$is a closed and convex set, and

$$
C_{k+}=p\left[\bar{N}\left(\operatorname{Con}(A, x), \varepsilon_{k}\right) \cap B_{+}\right]
$$

is a spherically convex set which contains no antipodal points. Similarly we define $C_{k-}$. Then $C_{k}=C_{k \div} \cup C_{k-}$ is a cell, contains $\chi(x)$ in its interior, and $\chi(x)=\bigcap_{k=1}^{\infty} C_{k}$.

From [2], Theorem 1 we conclude the existence of a map $g: S^{n} \rightarrow S^{n}$ with $g(x) \in S^{n} \backslash \chi(x)$ for all $x \in S^{n}$. Let $S^{n-1}(x)$ be the $(n-1)$-sphere obtained by intersecting $S^{n}$ with the hyperplane through 0 and orthogonal to $\overrightarrow{O x}$, and define $f: S^{n} \rightarrow S^{n}$ by taking as $f(x)$ the point of intersection of $S^{n-1}(x)$ with the semicircle from $x$ to $-x$ which contains $g(x)$. Clearly $f$ is continuous and is a rector field. As $f(x) \notin \varphi(x)$, a special homotopy from $\varphi$ to the vector field $-f$ can be constructed as in the proof of Lemma 2.5.

(ii) Now assume that the small multi-vector field $\varphi: S^{n} \rightarrow S^{n}$ is lsc. As $\varphi(x) \subset S^{n-1}(x)$, we see from Lemma 2.1 that $\operatorname{sconv} \varphi(x) \subset S^{n-1}(x)$. Hence the map $g: S^{n} \rightarrow S^{n}$ with $g(x) \in \operatorname{Scon} \nabla \varphi(x)$ provided by Proposition 3.1 is a vector field. The construction of the special homotopy $\Phi$ between $\varphi$ and $g$ in the proof of Lemma 2.5 is such that $\Phi$ is a homotopy between the vector fields $\varphi$ and $g$.

COROLLART 5.2. There exists no use or Isc small multi-vector field on $\mathbb{S}^{n}$ if $n$ is even. 


\section{References}

[1] C. Berge, Topological Spaces, Edinburgh and London 1963.

[2] T. R. Brahana, M. K. Fort, Jr., and Walt G. Horstman, Homotopy for cellular set-valued functions, Proc. Amer. Math. Soc. 16 (1965), pp. 455-459.

[3] L. Danzer, B. Grünbaum and V. Klee, Helly's theorem and its relatives, Proc. symposia in pure mathematics, vol. 7, Amer. Math. Soc., Providence, R. I. 1963.

[4] H. G. Eggleston Convexity, Cambridge Tracts in Mathematics and Mathematical Physics 47 (1966).

[5] J. W. Jaworowski, Some consequences of the Vietoris mapping theorem, Fund. Math. 45 (1958), pp. 261-272.

[6] E. Michael, Continuous selections I, Ann. of Math. 63 (1956), pp. 361-382.

[7] C. J. Rhee, Homotopy groups for cellular set-valued functions, Proc. Amer. Math. Soc. 19 (1968), pp. 874-876.

[8] H. Schirmer, $\delta$-continuous selections of small multifunctions, Canad. J. Math. 24 (1972), pp. 631-635.

[9] - Near fixed points of small usc multifunctions, to appear.

[10] W. L. Strother, Multi-homotopy, Duke Math. J. 22 (1955), pp. 281-285.

[11] F. A. Valentine, Convex Sets, New York, San Francisco, Toronto, London 1964

\section{CARLETON UNIVERSITY}

Ottawa

Recu par la Rédaction le 26, 1, 1972

\section{On a paper by Iqbalunnisa}

\section{by}

M. F. Janowitz (Amherst, Mass.)

Abstract. It is known that if $L$ is a complete lattice which is relatively complemented or (more generally) both section and dual section semicomplemented, then its congruence lattice is a Stone lattice. Recently, Iqbalunnisa has proved this to be true when $L$ is a complete, weakly modular, section complemented lattice. By weakening the axioms of weak modularity and section semicomplementation, a class of lattices is produced that includes all of the above examples, and for which the above result remains valid. A second class of lattices is then introduced on which a fairly explicit formula can be given for the pseudocomplement of a congruence relation. This second class includes all section semicomplemented lattices whose dual is section semicomplemented, and the formula for pseudocomplements is a new one for these lattices also.

1. Introduction. In [3], Theorem 2, p. 316, Iqbalunnisa proves that if $L$ is a complete, weakly modular, section complemented lattice, then the lattice of congruence relations of $L$ forms a Stone lattice, thus generalizing a result of the author ([5], Theorem 4.8, p. 202). On the other hand, the author has shown ([6], Theorem 4.17, p. 72) that if $L$ is a complete lattice which is both section semicomplemented and dual section semicomplemented, then its congruence lattice is a Stone lattice. Our purpose here is to provide a common generalization of these results. For convenience, our notation and terminology will follow that of [4]. Also, it will prove useful to let Axiom $\left(\mathrm{X}^{*}\right)$ denote the dual of Axiom (X) throughout the paper.

2. The general case. Though all of the above lattices are weakly modular, it turns out that we can get by with a slightly weaker axiom. Accordingly, we introduce Axiom (A) in a lattice with 0:

(A) $a / 0 \rightarrow c / d$ with $c>d$ implies $c / d \rightarrow a_{1} / a_{2}$ for suitable elements $a_{1}, a_{2}$ such that $a \geqslant a_{1}>a_{2}$.

LEMNA 1. Let $L$ be a lattice with 0. Axiom (A) is equivalent to the assertion that for every congruence relation $\Theta$ on $L, a \equiv 0\left(\Theta^{*}\right)$ iff the interval $[0, a]$ contains only trivial congruence classes modulo $\Theta$.

Proof. Let Axiom (A) hold. If $a \equiv 0\left(\Theta^{*}\right)$ and $a \geqslant b \geqslant c$ with $b \equiv c(\Theta)$, then $b \equiv c\left(\Theta \wedge \Theta^{*}\right)$ implies $b=c$. Suppose on the other hand 